\title{
A Complex Diffusion Driven Approach for Removing Data-Dependent Multiplicative Noise
}

\author{
P. Jidesh ${ }^{1}$ and A.A. Bini ${ }^{2}$ \\ 1 Department of Mathematical and Computational Sciences \\ 2 Department of Electronics and Communication Engineering, \\ National Institute of Technology, Karnataka, India-575025 \\ jidesh@nitk.ac.in, biniaa@gmail.com
}

\begin{abstract}
In this paper we propose a second-order non-linear PDE based on the complex diffusion function. The proposed method exhibits better restoration capability of ramp edges in comparison to other second-order methods discussed in the literature. The proposed model is designed for Gamma distributed multiplicative noise which commonly appears in Ultra Sound (US) and Synthetic Aperture Radar (SAR) images. The fidelity/reactive term augmented to the complex diffusive term is derived based on the Bayesian maximum a posteriori probability (MAP) estimator as detailed in Aubert and Ajol ([10]). The regularization parameter is selected based on the noise variance of the image and thus this adaptive method helps in restoring the images at various noise variances without manually fixing the parameter. The results shown in terms of both visual and qualitative measures demonstrate the capability of the model to restore images from their degraded observations.
\end{abstract}

Keywords: Complex diffusion, Multiplicative noise, Regularization method, Variational method.

\section{Introduction}

Restoration of images corrupted by data dependent multiplicative noise is a subject of practical interest in many imaging modalities. The data dependent multiplicative noise is commonly found in US and SAR images. Restoration under additive data dependent noise is quite extensively analyzed in the literature, the details can be found in Weickert ([3]) and the references therein. However, data dependent multiplicative noise is not so extensively analyzed in the literature. To our knowledge the first method of that kind was introduced by Rudin et al. ([5]). In this model the authors consider a data dependent multiplicative noise which follows a Gaussian distribution. This multiplicative Gaussian noise model is not of big practical interest. There are few dedicated studies those aim at restoring the images corrupted by Gamma and Poisson noise distributions, refer Aubert and Ajol ([10]) and Setzer et al.([12]) respectively, for further details. These restoration models discussed above use the Total Variation (TV) norm of the absolute gradient function as the functional to be minimized. The 
diffusion equation derived from the aforementioned functional gives a second order non-linear PDE. Quite a few second-order non-linear diffusion methods are also proposed for restoring images corrupted by the multiplicative noise, see $\mathrm{Yu}$ and $\operatorname{Acton}([4)$, Sun et al.([7]) and Krissian et al.([9]). These PDE models are variations of the well known Parona-Malik ([1]) model, where the coefficient of diffusion function is modified to handle various noise distributions. However these models do not directly derive the evolution equations from the noise model.

\section{Data-Dependent Multiplicative Noise Model}

Multiplicative noise commonly appears in many imaging modalities, especially in medical and satellite imaging. A common multiplicative noise model assumed in various restoration problems is defines as:

$$
u_{0}=u \times n,
$$

where $n$ is a multiplicative noise which has a mean 1 and variance $\sigma^{2}$. The first kind of restoration method proposed for this noise model is Rudin et al. ([5]). In this work the authors assume a Gaussian multiplicative noise. The gradient descent solution to the PDE proposed in this model is:

$$
u_{t}=\operatorname{div} \frac{\nabla u}{|\nabla u|}-\lambda \frac{u_{0}^{2}}{u^{3}}-\mu \frac{u_{0}}{u^{2}},
$$

where div is a divergence operator. Here the two Lagrange multipliers $\lambda$ and $\mu$ are dynamically updated to satisfy the constraints as explained in Rudin et al. ([5]). One of the major drawbacks of this model is that it assumes a Gaussian distribution for the noise. However, it is known from the history of image restoration that most of the imaging modalities of practical interest does not result in Gaussian distributed multiplicative noise. Here we assume a reflective boundary condition $(\partial u / \partial \boldsymbol{n}=0$, where $\boldsymbol{n}$ is a unit vector normal to the plane) and the initial condition $u(x, y, 0)=u_{0}(x, y)$, where $u_{0}(x, y)$ is the initial image. We further note that these boundary and initial conditions are assumed for all the PDEs stated in this work, if not mentioned otherwise.

There are few works those consider the multiplicative noise model as a log translated additive model, refer Krissian et al.(8]) for details. Here the authors assume the data dependent nature of the noise in the log compressed images. However one can easily note that even in log compressed images the noise does not become completely independent of data. Therefore these models fail to provide an effective restoration under the multiplicative noise setup.

In another work Aubert and Ajol([10]) modified the reactive term of the model in order to incorporate a Gamma distributive nature of the noise. This modified model is quite applicable to various ultrasound and SAR images, where the noise amplitude is Gamma distributed or the image is speckled. The evolution PDE can be stated as:

$$
u_{t}=\operatorname{div}\left(\frac{\nabla u}{|\nabla u|}\right)-\lambda \frac{\left(u-u_{0}\right)}{u^{2}} .
$$


Here $\lambda$ is a regularization parameter and other symbols have their usual meanings.

There are some modifications suggested for the above cited work, in the recent literature, see Huang et al.([13]) and Huang et al.([11]) for details. In Huang et al. ([13]) the authors replace the TV regularizer with a Weberized TV regularizer for a better restoration. And in Huang et al.([1] ) the authors provide a modified fitting term which is convex under all conditions, therefore the solution is unique.

Complex diffusion was introduced to image processing by Gilboa et al. in Gilboa et al. (6] ). A complex diffusion process motivated by the time dependent Schroedinger wave equation (a fundamental equation in quantum physics) can be written in an anisotropic manner as:

$$
u_{t}=\nabla \cdot c(\operatorname{Im}(u) \nabla u)
$$

where $\operatorname{Im}(u)$ denotes the imaginary component of complex image $u$ and

$$
c(\operatorname{Im}(u))=\frac{e^{i \theta}}{1+\left(\frac{\operatorname{Im}(u)}{k \theta}\right)^{2}},
$$

where $k$ is a threshold parameter. Further it was shown by the authors that the limiting case is $\lim _{\theta->0}(\mathrm{Im} / \theta)=t \Delta g_{\sigma} * u_{0}$, where $\Delta g_{\sigma} * u_{0}$ denotes the Gaussian convolved version of the Laplacian of the initial image. One can notice that since the coefficient of diffusion is a function of Laplacian of the image the ramp like edges are preserved more naturally in the filtered output, unlike other second-order non-linear diffusion methods.

\section{The Proposed Model}

In this work we propose a complex diffusion driven PDE for restoring specked images where the noise amplitude is Gamma distributed. In this proposed model the reactive term is borrowed from the multiplicative model proposed in Aubert and Ajol ([10] $)$. This reactive term is derived with regard to the noise distribution using the Bayesian maximum a posteriori probability estimator. The proposed diffusion model looks like

$$
u_{t}=\nabla \cdot(c(\operatorname{Im}(u)) \nabla u)-\lambda \frac{\left(u-u_{0}\right)}{u^{2}},
$$

where the symbols have the same meanings as defined earlier and function $c($. is defined in (5). Here $\lambda$ is a regularizing parameter which controls the diffusive and reactive functionalities of the filter. We assume the limiting case of the diffusion coefficient at small values of $\theta$. Since the negative diffusion leads to instability of the above PDE we assume the value of diffusion coefficient to be always positive. The initial condition $u(x, y, 0)=u_{0}(x, y)$ is assumed, where $u_{0}$ is the initial (observed) image which is real. One of the main features of complex diffusion is its capability to preserve ramp edges. It is observed that the gradient 
based diffusion coefficient functions are not quite effective in detecting the main features of ramps; namely its end points. Moreover these functions have nearly uniform values across the whole smoothed ramp, causing a non-linear gradientdependent diffusion to slow the diffusion process in those regions and therefore are less effective in noise reduction within the ramp-edges. However the Laplacian based diffusion coefficient functions have high magnitudes near the endpoints and low magnitudes elsewhere, and thus enables the nonlinear diffusion process to reduce noise over the ramp. Therefore, the proposed model is more effective in terms of noise reduction and ramp preservation compared to the other non-linear second-order diffusion processes like TV Rudin et al. ([2]) and Perona and Malik ([1]).

\subsection{Numerical Implementation}

We adopt an explicit time marching scheme for solving the proposed PDE numerically. The choice of the scheme is well justified by the reduced complexity and comparable accuracy. Here we show the discretization for the regularization term and the scheme is trivial for the reactive (fidelity) term.

$$
u^{n+1}=u^{n}+\Delta t\left(\frac{\partial^{-}}{\partial x}\left(\frac{\partial^{+} u}{\partial x} \times c(\operatorname{Im}(u))\right)+\frac{\partial^{-}}{\partial y}\left(\frac{\partial^{+} u}{\partial y} \times c(\operatorname{Im}(u))\right)\right),
$$

where $c($.$) is defined in (5) , u^{n}$ denotes the image at $n^{\text {th }}$ iteration and the super script $+/$ - denotes the forward/backward deference operators, respectively. The forward and backward difference operators along $x$ and $y$ are defined as:

$$
\begin{aligned}
& u_{x}^{+}=u(x+1, y)-u(x, y), u_{x}^{-}=u(x, y)-u(x-1, y), \\
& u_{y}^{+}=u(x, y+1)-u(x, y), u_{y}^{-}=u(x, y)-u(x, y-1) .
\end{aligned}
$$

Here we denote $\partial u / \partial x$ as $u_{x}$. Similarly we can derive $|\nabla u|$ as

$$
|\nabla u|=\sqrt{\left(\frac{\partial u}{\partial x}\right)^{2}+\left(\frac{\partial u}{\partial y}\right)^{2}}
$$

With the help of above discretization we can discretize the PDE in (6)

\section{Experimental Results and Discussions}

We have tested the efficiency of the proposed method using various test images, however due to the page limitations we have demonstrated the result only for the image "woman". The input images are normalized to the range [0-1] in terms of their intensities. The Gamma noise is generated using a function written in Matlab. We have tested the images under various noise densities and the proposed model is found to be better in terms of noise reduction and details preservation as compared to the other methods considered in this work. A quantitative study is 

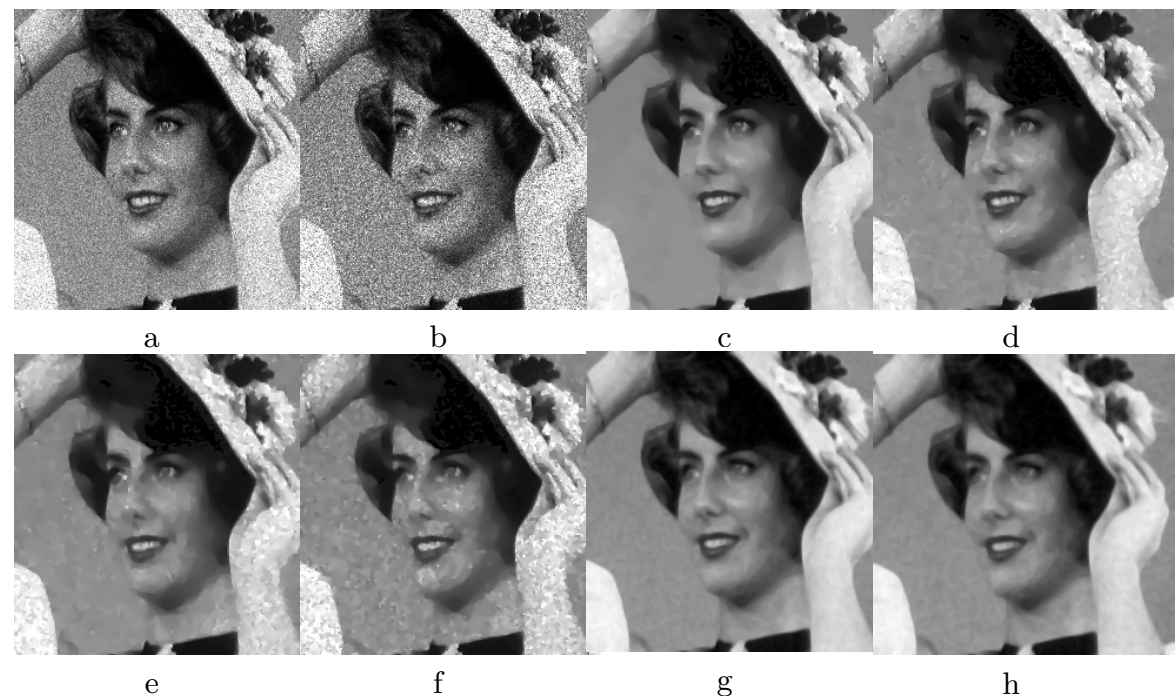

Fig. 1. Images restored using various methods: (a) and (b): Noisy images with mean 1 and noise variances 0.1 and 0.2 , respectively; (c)and (d) restored using AA method; (e) and (f) restored using RLO filter; (g) and (h) restored using the proposed method

carried out using the statistical measure Peak Signal to noise Ratio (PSNR) and the results are provided in Table 1. Figure 1 shows the output of various methods considered in this work. The existing filters considered here for comparison are Aubert Ajol ([10]) filter and TV filter for multiplicative noise by Rudin et al. ([5]). From the results provided in Figure 1 one can confirm that the proposed method outperforms other methods in terms of visual appearance. Moreover the Peak Signal to Noise Ratio tabulated in Table 1, provides an understanding on the noise reduction capacity of various methods under consideration, tested with different images at a specified noise variance. The proposed method shows a better PSNR value compared to other considered methods. In addition to this the number of iterations taken by various methods to reach at the steady state was also analyzed in this study. We confirm that the proposed model takes slightly

Table 1. PSNR evaluated for various restoration methods for different images. (The gamma noise variance of the input image is :0.2).

\begin{tabular}{ccccc}
\hline Image Name Noisy & RLO & AA & The proposed model \\
\hline woman & 11.27 & 12.91 & 15.27 & 16.63 \\
cameraman & 12.12 & 14.81 & 16.12 & 16.91 \\
Lena & 11.32 & 13.41 & 14.12 & 15.91 \\
peppers & 12.33 & 13.22 & 14.67 & 15.47 \\
\hline
\end{tabular}


more number of iterations to reach the steady state in comparison to AA and RLO models, at different noise density levels.

\section{Concluding Remarks}

In this paper we have proposed a complex diffusion driven regularization filter for restoring images corrupted by multiplicative noise whose amplitude is Gamma distributed (speckled images). The restoration is found to preserve ramp features present in images. The experimental results prove beyond any doubts that the proposed model performs better in terms of visual and quantitative measures provided in the result section.

\section{References}

1. Perona, P., Malik, J.: Scale-space and edge detection using anisotropic diffusion. IEEE Transactions on Pattern Analysis and Machine Intelligence 12, 629-639 (1990)

2. Rudin, L.I., Osher, S., Fehtami, E.: Nonlinear total variation based noise removal algorithms. Physica D. 68, 259-268 (1992)

3. Weickert, J.: A Review of Nonlinear Diffusion Filtering Scale-Space, 3-28 (1997)

4. Yu, Y., Acton, S.T.: Speckle reducing anisotropic diffusion. IEEE Transactions on Image Processing 11, 1260-1270 (2002)

5. Rudin, L.I., Lions, P.L., Osher, S.: Multiplicative denoising and deblurring: theory and algorithms. In: Geometric Level Set Methods in Imaging, Vision, and Graphics, pp. 103-120. Springer, Berlin (2003)

6. Gilboa, G., Sochen, N.A., Zeevi, Y.V.: Image Enhancement and Denoising by Complex Diffusion Processes. IEEE Trans. Pattern Anal. Mach. Intell. 26, 1020-1036 (2004)

7. Sun, Q., Hossack, J.A., Tang, J., Acton, S.T.: Speckle reducing anisotropic diffusion for 3D ultrasound images. Computerized Medical Imaging and Graphics 28, 461-470 (2004)

8. Krissian, K., Kikinis, R., Westin, C.F., Vosburgh, K.: Speckle constrained filtering of ultrasound images. Proceedings of IEEE Comput. Vis. Pattern Recogn. (CVPR), 547-552 (2005)

9. Krissian, K., Westin, C.F., Kikinis, R., Vosburgh, K.G.: Oriented speckle reducing anisotropic diffusion. IEEE Transactions on Image Processing 16, 1412-1424 (2007)

10. Aubertt, G., Aujol, J.F.: A variational approach to removing multiplicative noise. SIAM Journal on Applied Mathematics 68, 925-946 (2008)

11. Huang, M., Ng, M.K., Wen, Y.: A New Total Variation Method for Multiplicative Noise Removal. SIAM J. Imaging Sciences 2, 22-40 (2009)

12. Setzer, S., Steidl, G., Teuber, T.: Deblurring Poissonian Images by Split Bregman Techniques. J. Visual Communication and Image Representation 21, 193-199 (2010)

13. Huang, L., Xiao, L., Wei, Z.-H.: Multiplicative Noise Removal via a Novel Variational Model. EURASIP Journal on Image and Video Processing 2, 1-16 (2010) 